(c) Elsevier/Inra

Original article

\title{
Restricted multistage selection indices
}

\author{
C Xie, S Xu \\ Department of Botany and Plant Sciences, University of California, \\ Riverside, CA 92521-0124, USA
}

(Received 12 November 1996; accepted 3 April 1997)

\begin{abstract}
Summary - Multistage selection index is an important extension to single stage selection index for genetic improvement of multiple traits. Recently, a multistage selection procedure, referred to as selection index updating, has been developed. This procedure combines several desired aspects of independent culling and selection index. In this study, we directly extend selection index updating to cases of restricted multistage selection indices by imposing restrictions for solving index coefficients. The resulting indices restrict genetic changes to zero or to some proportion in the chosen characters or linear functions of characters. As such, it makes multistage selection indices more flexible. The possibility of imposing restrictions on different stages is also discussed. A numerical example is given to illustrate the calculation of restricted multistage selection indices.
\end{abstract}

independent culling / multistage selection / restricted selection index / sequential selection

Résumé - Index contraints dans la sélection à étapes. L'index de sélection à plusieurs étapes est une extension importante de l'index de sélection à une seule étape. Récemment, $X u$ et Muir ont développé une procédure de sélection à plusieurs étapes appelée index de sélection avec mise $\grave{i}$ jour. Cette procédure combine plusieurs aspects intéressants de la sélection sur index et de la sélection à niveaux indépendants. Dans cette étude, nous étendons directement la mise à jour des index de sélection au cas d'index de sélection à plusieurs étapes en imposant des restrictions pour trouver les coefficients des index. Les index correspondants contraignent les progrès génétiques à être nuls ou à être dans des rapports donnés pour des caractères ou des combinaisons de caractères. De la sorte, les index de sélection multiétapes sont plus souples. La possibilité d'imposer des restrictions $\grave{a}$ différentes étapes est aussi discutée. Un exemple numérique est donné pour illustrer le calcul des index de sélection multiétapes contraints.

sélection à niveaux indépendants / sélection multiétapes / index de sélection contraint / sélection séquentielle

\section{INTRODUCTION}

The genetic merit of a plant or an animal is often defined as a function of several traits, and is predicted by an index that combines information on its own and relatives' measurements. Smith (1936) and Hazel (1943) presented a linear index 
that maximizes the improvement of an overall genetic value for all traits. Later, Kempthorne and Nordskog (1959) introduced the idea of restricted selection index, which holds certain characters constant while allowing other characters to increase freely. Tallis (1962) and Harville (1975) extended this idea to the case where some traits can be changed by amounts proportional to a predetermined selection goal. Rao (1962) presented a method for computing an index to improve one trait while requiring changes in other traits to be in specific directions. James (1968) showed how restrictions could simultaneously be imposed on the genetic results of selection and on the index weighting factors. The theory of restricted selection indexes was further extended by Niebel and Van Vleck (1982). They introduced fixed and proportional restrictions when more than one selection index is used in a population. A detailed review of restricted selection indices was given by Brascamp (1984).

When traits have a developmental sequence in ontogeny or there is a large difference in the cost of measuring various traits, independent culling or multistage index selection for multiple trait genetic improvements is the most efficient procedure with respect to cost saving owing to the possibility of early culling (Young and Weiler, 1960; Young, 1964; Namkoong, 1970; Cunningham, 1975; Ducrocq and Colleau, 1989; Norell et al, 1991). In most cases, the extension of these developments for independent culling selection to the case of selecting combination of traits at each stage is straightforward. Recently, Xu and Muir $(1991,1992)$ developed a multistage selection procedure, referred to as selection index updating, that combines several desired aspects of independent culling and selection index. Use of selection index updating allows breeders to determine the optimum truncation points for the maximization of genetic gain in aggregate breeding value, economic return in terms of genetic gain per unit cost, or profit.

In all multistage selection procedures presented by those authors, the selection goal was to improve all characters without restrictions. However, a breeder may be interested in increasing the overall genetic gain with restrictions that certain traits remain constant or change in a predetermined amount. For instance, a poultry breeder may feel that mean egg size should be kept at a constant intermediate level while using a multistage selection index to maximize overall economic value. The breeder may manage this based on body weight and egg weight measured at the first stage, and production at the second stage. Hence, a two-stage restricted selection index may arise in practice. The purpose of the present note is to extend restricted single stage selection indices to the case of restricted multistage selection indices.

\section{THEORY}

In subsequent derivations the assumptions and notation will be similar to those of $\mathrm{Xu}$ and Muir (1992). For convenience, the relevant notation is summarized below:

$\mathbf{y}=$ an $n \times 1$ vector of phenotypic values of $n$ traits with elements $y_{i}$,

$\mathbf{g}=\mathrm{a} k \times 1$ vector of genetic values of $k$ traits to be improved with elements $g_{i}$,

$\mathbf{w}=\mathbf{a} k \times 1$ vector of economic weights with elements $w_{j}$,

$Z=\mathbf{b}^{T} \mathbf{y}$, selection index, where the superscript $T$ denotes matrix transposition, 
$H=\mathbf{w}^{T} \mathbf{g}$, the aggregate breeding value,

$\mathbf{P}=\operatorname{Var}(\mathbf{y})$, an $n \times n$ phenotypic variance-covariance matrix,

$\mathbf{G}=\operatorname{Cov}\left(\mathbf{y}, \mathbf{g}^{T}\right)$, an $n \times k$ genetic covariance matrix, and

$\mathbf{G}_{r}=$ an $n \times r$ matrix corresponding to the $r$ columns (restrictions) of $\mathbf{G}$.

Suppose that $n$ traits are to be selected in $m$ stages $(m<n)$. The phenotypic values of $n$ traits, $\mathbf{y}$, can be partitioned into $m$ subvectors, $\mathbf{y}_{1}, \mathbf{y}_{2} \ldots \mathbf{y}_{m}$. For example, if there are four traits to be selected in three stages, $y_{1}$ and $y_{2}$ are measured in the first stage, $y_{3}$ is obtained in the second stage, and $y_{4}$ is measured in the third stage, then $\mathbf{y}=\left[\begin{array}{llll}y_{1} & y_{2} & y_{3} & y_{4}\end{array}\right]^{T}, \mathbf{y}_{1}=\left[\begin{array}{ll}y_{1} & y_{2}\end{array}\right]^{T}, \mathbf{y}_{2}=\left[\begin{array}{lll}y_{1} & y_{2} & y_{3}\end{array}\right]^{T}$,

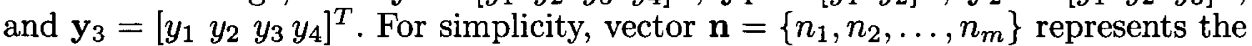
number of traits measured up to stage $m$, where $n_{i}$ denotes the number of traits measured up to the $i$ th stage. Let $\mathbf{z}=\left[\begin{array}{lll}Z_{1} & Z_{2} & Z_{3}\end{array}\right]^{T}$ be a $3 \times 1$ vector of the updated selection indices defined by $\mathrm{Xu}$ and Muir (1992), then the indices for the above example are

$$
\mathbf{z}=\mathbf{B}^{T} \mathbf{y}
$$

where

$$
\mathbf{B}=\left[\begin{array}{ccc}
b_{11} & b_{12} & b_{13} \\
b_{21} & b_{22} & b_{23} \\
0 & b_{32} & b_{33} \\
0 & 0 & b_{43}
\end{array}\right]
$$

is a $4 \times 3$ matrix with the $i$ th column, $\mathbf{b}_{i}$, representing the weights for the selection indices at the $i$ th stage. Thus, the selection index at the $i$ th stage is

$$
Z_{i}=\mathbf{b}_{i}^{T} \mathbf{y}_{i}, i=1,2, \ldots, m
$$

According to $\mathbf{y}_{1}, \mathbf{y}_{2}, \ldots, \mathbf{y}_{m}, \mathbf{P}$ and $\mathbf{G}$ can be correspondingly partitioned as:

$$
\mathbf{P}=\left[\begin{array}{cccc}
\mathbf{P}_{11} & \mathbf{P}_{12} & \ldots & \mathbf{P}_{1 m} \\
\mathbf{P}_{21} & P_{22} & \ldots & \mathbf{P}_{2 m} \\
& \vdots & & \vdots \\
\mathbf{P}_{m 1} & \mathbf{P}_{m 2} & \ldots & \mathbf{P}_{m m}
\end{array}\right] \text { and } \mathbf{G}=\left[\begin{array}{c}
\mathbf{G}_{1} \\
\mathbf{G}_{2} \\
\vdots \\
\mathbf{G}_{m}
\end{array}\right]
$$

Let $\mathbf{Q}$ be a submatrix of $\mathbf{P}$ and $\mathbf{A}$ be a submatrix of $\mathbf{G}$, ie, $\mathbf{Q}_{i j}=[\mathbf{P}]_{i x j}$ and $\mathbf{A}_{i}=[\mathbf{G}]_{i}$ for $i, j=1,2, \ldots, m$. Note that $[\mathbf{G}]_{i}$ differs from $\mathbf{G}_{r}$ defined before. Selection response based on an index that maximizes the economic values of selected individuals is proportional to $\rho_{Z H}$ (Kempthorne and Nordskog, 1959), the correlation between index $\left(Z_{i}\right)$ values and genetic merits $\left(H=\mathbf{w}^{T} \mathbf{g}\right)$, that is

$$
\rho_{Z H}^{2}=\frac{\operatorname{Cov}^{2}\left(Z_{i}, H\right)}{\sigma_{Z}^{2} \sigma_{H}^{2}}=\frac{\left(\mathbf{w}^{T} \mathbf{A}_{i}^{T} \mathbf{b}_{i}\right)^{2}}{\mathbf{b}^{T} \mathbf{Q}_{i i} \mathbf{b}_{i} \mathbf{w}^{T} \mathbf{A} \mathbf{w}}
$$

where $\mathbf{A}$ is the covariance matrix of genetic values of $k$ traits. The $\mathbf{b}_{i}$ s are found such that $\rho_{Z H}$ is a maximum or equivalently

$$
E\left[\left(\mathbf{b}_{i}^{T} \mathbf{y}_{i}-\mathbf{w}^{T} \mathbf{g}\right)^{T}\left(\mathbf{b}_{i}^{T} \mathbf{y}_{i}-\mathbf{w}^{T} \mathbf{g}\right)\right]
$$


is a minimum subject to the restrictions

$$
\begin{aligned}
& \operatorname{Cov}\left(\mathbf{g}_{r}, Z_{i}\right)=\mathbf{G}_{r}^{T} \mathbf{b}_{i}=\mathbf{k}_{r} \\
& \operatorname{Cov}\left(Z_{i}, \mathbf{z}_{(i-1)}\right)=\mathbf{b}_{i}^{T} \mathbf{Q}_{i(i-1)} \mathbf{B}_{(i-1)}=\mathbf{0}
\end{aligned}
$$

where $\mathbf{g}_{r}$ is a $r \times 1$ vector of genetic values for $r$ restricted traits, $\mathbf{k}_{r}$ is a $r \times 1$ vector of desired values, $\mathbf{z}_{(i-1)}=\left[Z_{1}, \ldots, Z_{(i-1)}\right]$ is a vector of selection indices, and $\mathbf{B}_{(i-1)}$ is a submatrix of $B$ that contains index coefficients up to stage $i-1$. To insure there exists a solution, it has to be that $r<n_{i}$, ie, the number of restrictions at a given stage should be less than the number of characters at the same stage. The first restriction [6] constrains some characters to be changed in predetermined amounts (Tallis, 1962). The second restriction [7], requiring that indices at different stages are independent, insures the existence of an exact solution for truncation points without resorting to multiple integration (Xu and Muir, 1991, 1992).

After introducing Lagrange multipliers of $\lambda$ and $\tau$ ( $\lambda$ is an $r$ vector and $\tau$ is an $m-1$ vector), the optimum $\mathbf{b}_{i}$ is found by minimizing the following quantity with respect to $\mathbf{b}_{i}$ :

$$
\mathbf{T}=\mathbf{b}_{i}^{T} \mathbf{Q}_{i i} \mathbf{b}_{i}-2 \mathbf{b}_{i}^{T} \mathbf{A}_{i} \mathbf{w}+\mathbf{w}^{T} \mathbf{A} \mathbf{w}-2 \lambda^{T}\left(\mathbf{G}_{r} \mathbf{b}_{i}-\mathbf{k}_{r}\right)-2 \tau^{T}\left(\mathbf{b}_{i}^{T} \mathbf{Q}_{i(i-1)} \mathbf{B}_{(i-1)}\right)[8]
$$

Vector differentiation gives

$$
\partial \mathbf{T} / \partial \mathbf{b}_{i}=2 \mathbf{b}_{i}^{T} \mathbf{Q}_{i i}-2 \mathbf{w}^{T} \mathbf{A}_{i}-2 \lambda^{T} \mathbf{G}_{r}-2 \tau^{T} \mathbf{B}_{(i-1)}^{T} \mathbf{Q}_{(i-1) i}=\mathbf{0}
$$

or

$$
\mathbf{b}_{i}=\mathbf{Q}_{i i}^{-1}\left(\mathbf{G}_{r}^{T} \lambda+\mathbf{R}_{i(i-1)}^{T} \tau+\mathbf{w}^{T} \mathbf{A}_{i}\right)
$$

where $\mathbf{R}_{i(i-1)}=\mathbf{B}_{(i-1)}^{T} \mathbf{Q}_{(i-1) i}$. From constraints [6] and [7], we obtain

$$
\begin{gathered}
\mathbf{G}_{r} \mathbf{Q}_{i i}^{-1}\left(\mathbf{G}_{r}^{T} \lambda+\mathbf{R}_{i(i-1)}^{T} \tau+\mathbf{w}^{T} \mathbf{A}_{i}\right)-\mathbf{k}_{r}=\mathbf{0} \\
\mathbf{R}_{(i-1) i} \mathbf{Q}_{i i}^{-1}\left(\mathbf{G}_{r}^{T} \lambda+\mathbf{R}_{i(i-1)}^{T} \tau+\mathbf{w}^{T} \mathbf{A}_{i}\right)=\mathbf{0}
\end{gathered}
$$

where $\mathbf{R}_{(i-1) i}=\mathbf{Q}_{i(i-1)} \mathbf{B}_{(i-1)}=\mathbf{R}_{i(i-1)}^{T}$. After solving equations [11a, b] for $\lambda$ and $\tau$ and substituting them into [10], we have

$$
\begin{gathered}
\mathbf{b}_{i}=\left\{\left(\mathbf{I}-\mathbf{Q}_{i i}^{-1} \mathbf{M}_{i}\right)\left[\mathbf{I}-\mathbf{Q}_{i i}^{-1} \mathbf{G}_{r}^{T} \mathbf{H}_{i}^{-1} \mathbf{G}_{r}\left(\mathbf{I}-\mathbf{Q}_{i i}^{-1} \mathbf{M}_{i}\right)\right]\right\} \mathbf{Q}_{i i}^{-1} \mathbf{A}_{i} \mathbf{w} \\
+\left(\mathbf{I}-\mathbf{Q}_{i i}^{-1} \mathbf{M}_{i} \mathbf{Q}_{i i}^{-1}\right) \mathbf{G}_{r}^{T} \mathbf{H}_{i}^{-1} \mathbf{k}_{r}
\end{gathered}
$$

where $\mathbf{I}$ is an identity matrix with the same dimension as $\mathbf{Q}_{i i}$, and

and

$$
\begin{aligned}
\mathbf{M}_{i} & =\mathbf{R}_{i(i-1)}\left[\mathbf{R}_{(i-1) i} \mathbf{Q}_{i i}^{-1} \mathbf{R}_{i(i-1)}\right]^{-1} \mathbf{R}_{(i-1) i} \\
\mathbf{H}_{i} & =\mathbf{G}_{r} \mathbf{Q}_{i i}^{-1} \mathbf{G}_{r}^{T}-\mathbf{G}_{r} \mathbf{Q}_{i i}^{-1} \mathbf{M}_{i} \mathbf{Q}_{i i}^{-1} \mathbf{G}_{r}^{T}
\end{aligned}
$$

Equation [12] is the solution for coefficients for a restricted multistage selection index. It has a form similar to the optimal single stage selection index of Tallis (1962). Note that when $\mathbf{k}_{r}=\mathbf{0}$, the above expression reduces to:

$$
\mathbf{b}_{i}=\left\{\left(\mathbf{I}-\mathbf{Q}_{i i}^{-1} \mathbf{M}_{i}\right)\left[\mathbf{I}-\mathbf{Q}_{i i}^{-1} \mathbf{G}_{r}^{T} \mathbf{H}_{i}^{-1} \mathbf{G}_{r}\left(\mathbf{I}-\mathbf{Q}_{i i}^{-1} \mathbf{M}_{i}\right)\right]\right\} \mathbf{Q}_{i i}^{-1} \mathbf{A}_{i} \mathbf{w}
$$


This is similar to the restricted single stage selection index of Kempthorne and Nordskog (1959). When $r=0$, ie, $\mathbf{G}_{r}=\mathbf{0}$, equation [14] gives the ordinary unrestricted multistage selection index presented by Xu and Muir (1992)

$$
\mathbf{b}_{i}=\left(\mathbf{I}-\mathbf{Q}_{i i}^{-1} \mathbf{M}_{i}\right) \mathbf{Q}_{i i}^{-1} \mathbf{A}_{i} \mathbf{w}
$$

Let $\Delta z_{i}$ be the coefficient of standardized selection intensity for the $i$ th selection index and $\Delta z=\left[\Delta z_{1} \ldots \Delta z_{i} \ldots \Delta z_{m}\right]$, then the vector of genetic gains, $\Delta \mathbf{G}$, is predicted by

$$
\Delta \mathbf{G}=\left[\Delta G_{1} \ldots \Delta G_{k}\right]^{T}=\mathbf{G}^{T} \mathbf{B} \Delta \mathbf{z}
$$

where $\Delta G_{i}$, the $i$ th element of $\Delta \mathbf{G}$, is the genetic gain of the $i$ th trait. The total genetic change in aggregate breeding value is

$$
\Delta H=\sum_{i=1}^{m} \mathbf{w} \mathbf{A}_{i}^{T} \mathbf{b}_{i} \Delta z_{i}
$$

where $\mathbf{w}$ are the economic values of the traits, and the $\mathbf{A}_{i}$ is defined as before.

Note that equation [17] provides the linear relationship between $\Delta H$ and $\Delta \mathbf{z}$, but does not indicate how to select the optimal set of $\Delta \mathbf{z}$ so that $\Delta H$ is maximized. Let $u_{i}$ be the truncation point corresponding to selection intensity $\Delta z_{i}$ and $q_{i}=1-\Phi\left(u_{i}\right)$ be the proportion selected for the $i$ th index, where $\Phi$ is the cumulative distribution function of the standard normal distribution. The interrelationship among $\Delta z_{i}, u_{i}$ and $q_{i}$ is

$$
\Delta z_{i}=\frac{\exp \left\{-\frac{1}{2} u_{i}^{2}\right\}}{q_{i} \sqrt{2 \pi}}
$$

Substituting equation [18] into [17] and using Newton's method, the optimum $\Delta H$ can be found by maximizing $\Delta H$ with respect to $\mathbf{u}=\left[\begin{array}{ll}u_{1} & u_{2} \ldots u_{m}\end{array}\right]^{T}$ under the constraint

$$
\prod_{j=1}^{m} q_{j}=p
$$

where $p$ is a predetermined total proportion selected (Xu and Muir, 1992; Xu et al, 1995).

\section{NUMERICAL EXAMPLE}

Data from a classical example of index selection given by Hazel (1943) and Cunningham (1975) are used to illustrate the computation of restricted multistage selection indices. The information used in a swine selection scheme comprises the five traits: pig's own market weight $\left(y_{1}\right)$, pig's own market score $\left(y_{2}\right)$, productivity of dam $\left(y_{3}\right)$, average market weight of pig and littermates $\left(y_{4}\right)$, and average market score of pig and littermates $\left(y_{5}\right)$. Only the first three traits are assumed to be economically important (by Hazel, 1943; Cunningham, 1975). The economic weights 
are $\mathbf{w}=[1 / 3,1,2]$. The estimated phenotypic and genotypic variance-covariance matrices for the five traits are:

$$
\begin{aligned}
& \begin{array}{llllll}
y_{1} & y_{2} & y_{3} & y_{4} & y_{5}
\end{array} \\
& \mathbf{P}=\left[\begin{array}{rrrrr}
1015.0596 & 93.5066 & -7.4323 & 457.9949 & 41.2218 \\
93.5066 & 22.8484 & -3.7634 & 41.2218 & 8.2985 \\
-7.4323 & -3.7634 & 94.4784 & -7.4323 & -3.7634 \\
457.9949 & 41.2218 & -7.4323 & 457.9949 & 41.2218 \\
41.2218 & 8.2985 & -3.7634 & 41.2218 & 8.2985
\end{array}\right]
\end{aligned}
$$

and

$$
\mathbf{G}=\left[\begin{array}{rrr}
302.5893 & 13.5093 & 0.0 \\
13.5093 & 2.2391 & 0.0 \\
0.0 & 0.0 & 7.6339 \\
181.5536 & 8.1056 & 0.0 \\
8.1056 & 1.3435 & 0.0
\end{array}\right]
$$

The overall proportion selected is set to $p=6 \%$, having a standardized selection differential, $\Delta z=1.98538$.

A three-stage selection is performed with $y_{1}$ and $y_{2}$ selected at the first stage, $y_{3}$ at the second stage, and $y_{4}$ and $y_{5}$ at the third stage. This selection procedure is simply denoted as $\mathbf{n}=\{2,3,5\}$. We now assume that the selection goal is to keep $y_{2}$ constant or change at a desired level, while allowing $y_{1}$ and $y_{3}$ to increase without constraints. The unrestricted three-stage selection has index coefficients that are determined by equation [15], ie,

$$
\left[\begin{array}{ccc}
\mathbf{b}_{1} & \mathbf{b}_{2} & \mathbf{b}_{3} \\
0.03681 & -0.00130 & -0.03259 \\
-0.07149 & 0.02234 & 0.05703 \\
0 & 0.10328 & -0.00092 \\
0 & 0 & 0.07540 \\
0 & 0 & -0.16477
\end{array}\right]
$$

A multidimensional Newton's iterative equation system is used to search for the optimal truncation points $\left(u_{i} \mathrm{~s}\right)$ (Xu and Muir, 1992). The sets of truncation points $\left(u_{i}\right)$, standardized selection differentials $\left(\Delta z_{i}\right)$, and the proportions selected in each stage $\left(q_{i}\right)$ are:

$$
\begin{aligned}
& \mathbf{u}=\left[\begin{array}{rrr}
1.34806 & -0.66292 & -1.31152
\end{array}\right]^{T} \\
& \Delta \mathbf{z}=\left[\begin{array}{lrr}
1.81045 & 0.42911 & 0.18650
\end{array}\right]^{T} \\
& \mathbf{q}=\left[\begin{array}{lll}
0.08882 & 0.74631 & 0.90516
\end{array}\right]^{T}
\end{aligned}
$$

The gains for three traits predicted from this index are $\Delta \mathbf{G}=\left[\begin{array}{ll}18.98569 & 0.63881\end{array}\right.$ $0.33699]^{T}$ and the aggregate economic value is $\Delta H=7.64128$. 
Assume now that we want to maximize the response from selection with the restriction of no change $\left(k_{y 2}=0\right)$ in attribute $y_{2}$, where the subscript $y_{2}$ is used to denote the corresponding values for the second trait $\left(y_{2}\right)$ and the same thereafter. For this case, $\mathbf{G}_{y 2}=\left[\begin{array}{lllll}13.5093 & 2.2391 & 0.0000 & 8.1056 & 1.3435\end{array}\right]^{T}$. That is, $\mathbf{G}_{y 2}$ consists of the second column of $\mathbf{G}$. The restricted index coefficients computed from equation [14] are:

$$
\left[\begin{array}{ccr}
\mathbf{b}_{1} & \mathbf{b}_{2} & \multicolumn{1}{c}{\mathbf{b}_{3}} \\
0.03731 & -0.00219 & -0.02355 \\
-0.22509 & 0.01322 & 0.19453 \\
0 & 0.10306 & -0.00904 \\
0 & 0 & 0.07086 \\
0 & 0 & -0.51489
\end{array}\right]
$$

Using the same sets of $u_{i} \mathrm{~s}, \Delta z_{i}$ s and $q_{i}$ s as those obtained from the unrestricted selection indices, we obtain genetic gains, $\Delta \mathbf{G}=\left[\begin{array}{lll}15.5069 & 0.0000 & 0.3247\end{array}\right]^{T}$, and aggregate economic value, $\Delta H=5.81835$. Therefore, selection on restricted multistage indices should result in zero gain in $y_{2}$ as expected. As a further check, it can be verified that $\operatorname{Cov}\left(g_{y 2}, Z_{i}\right)=\mathbf{G}_{y 2}^{T} \mathbf{b}_{2}=0$, when $k_{y 2}=0$.

As a final example, we demonstrate an index that maximizes economic gain while allowing $y_{2}$ to reach a predetermined value (Tallis, 1962, 1968). Let $\operatorname{Cov}\left(g_{y 2}, Z_{i}\right)=$ -0.2 . Then the restricted selection procedure has index coefficients,

$$
\left[\begin{array}{ccc}
\mathbf{b}_{1} & \mathbf{b}_{2} & \mathbf{b}_{3} \\
0.03493 & -0.03098 & -0.00026 \\
-0.24292 & -0.00440 & 0.00099 \\
0 & 0.00027 & -0.00647 \\
0 & 0 & -0.04539 \\
0 & 0 & 0.01312
\end{array}\right]
$$

with $\Delta \mathbf{G}=\left[\begin{array}{lll}7.58025 & -0.38624 & -0.00831\end{array}\right]^{T}$ and $\Delta H=2.12386$.

A summary of genetic gains and aggregate breeding values from single- or multistage selection with or without restrictions is shown in table I. The genetic gain from one-stage selection is used as a standard for comparing the two- or three-stage selection procedure. The unrestricted two-stage selection presented here is the same as Cunningham's selection procedure 4 except that the actual truncation point in the first stage selection is predetermined in Cunningham's procedure $4(u=1.2338)$ with a proportion selected on $I_{1}$ of $38 \%$ and is optimized in selection index updating $\left(u=1.5091\right.$ ) with a proportion selected on $Z_{1}$ of $6.56 \%$. This leads to the aggregate breeding value of 8.0773 in this note and of 7.8675 presented by Cunningham (1975).

For a given stage, the index is computed from $Z_{i}=\mathbf{b}_{i}^{T} \mathbf{y}_{i}$. As selection stages advance, more and more information is incorporated into the index. In practice, it is necessary that restrictions on some characters should be incorporated into the index at a later stage when the trait measurements become available. This problem is solved by modifying $\mathbf{G}_{r}$ and $\mathbf{k}_{r}$ of equation [12] for each stage of selection since 
the index is derived with $\operatorname{Cov}\left(Z_{i}, Z_{j}\right)=0$ for $i \neq j$. For example, suppose we desired no genetic changes for $y_{2}$ and $y_{3}$ in a three-stage selection with $\mathbf{n}=\{2,4,5\}$. Thus, the constraint on $y_{2}$ would begin at the first stage and would begin on $y_{3}$ at the second stage. Under such a selection procedure, the restricted selection indices give genetic gains of $\Delta \mathbf{G}=\left[\begin{array}{lll}15.28095 & 0.0000 & 0.0000\end{array}\right]^{T}$ whereas the unrestricted selection indices produce genetic gains of $\Delta \mathbf{G}=\left[\begin{array}{lll}18.90569 & 0.65909 & 0.41079\end{array}\right]^{T}$. From this it can be seen that selection on restricted multistage indices where restrictions are incorporated into index at different stages results in zero gains as expected.

\section{DISCUSSION}

There are a number of practical situations to which the techniques of the previous sections could be applied. For example, in the selection of dual-purpose bulls for use in artificial insemination, the bulls are first selected using information from their parents; they are subsequently screened for their own performance, and finally selected on the basis of a progeny test of their daughters. In that case, selection can be regarded as a stochastic process in continuous time. For practical and economic reasons the selection is performed in several stages in such situations (Cunningham, 1975; Niebel and Van Vleck, 1982; Norell et al, 1991; Xu et al, 1995).

When selection uses all information collected from a number of traits performed in several stages, the selection objective usually remains constant. That is, we are interested in the quantity associated with primary product. In some situations, certain growth patterns may be more economical, or otherwise more desirable, than others. For instance, in the example given by Tallis (1968) one desirable characteristic in fat lamb production is rapid and early increase in body weight. On the other hand, if the body weight increases too much, some production and marketing problems may arise. Hence, selection is a process to use available information to push breeds towards the optimum growth pattern (Tallis, 1968; Harville, 1975; Niebel and Van Vleck, 1982). The technique of restricted selection index is justified as a means to achieve a prespecified selection goal (Lin, 1989).

The procedure given here either restricts genetic changes to zero or to proportional changes in chosen characters or linear functions of characters. In this note, the restrictions are achieved by calculating $\mathbf{b}_{i}$ under certain constraints and are unrelated to economic weighting factors. The restrictions also might be achieved by modifying economic weighting factors (James, 1968; Lin, 1989).

The effect of restricted multistage selection indices is similar to restricted single stage selection indices. For instance, the restriction is obviously achieved at the expense of total aggregate genetic gain as compared with unrestricted indices (table I). Selection on restricted multistage indices produces an even lower gain than that on restricted single stage index. This is similar to cases of unrestricted selection where the multistage selection index would result in less genetic gain than the single stage selection index owing to early culling (Xu and Muir, 1991). However, reduction in genetic gains, as one referee pointed out, is not necessarily coupled with restricted index selection. Because economic weights in the numerical example are assumed to be known, then one of the traits was restricted to a less optimum level. As such, genetic gain for the restricted index is significantly less than that obtained with the optimum index (table I). Nevertheless, in many situations 


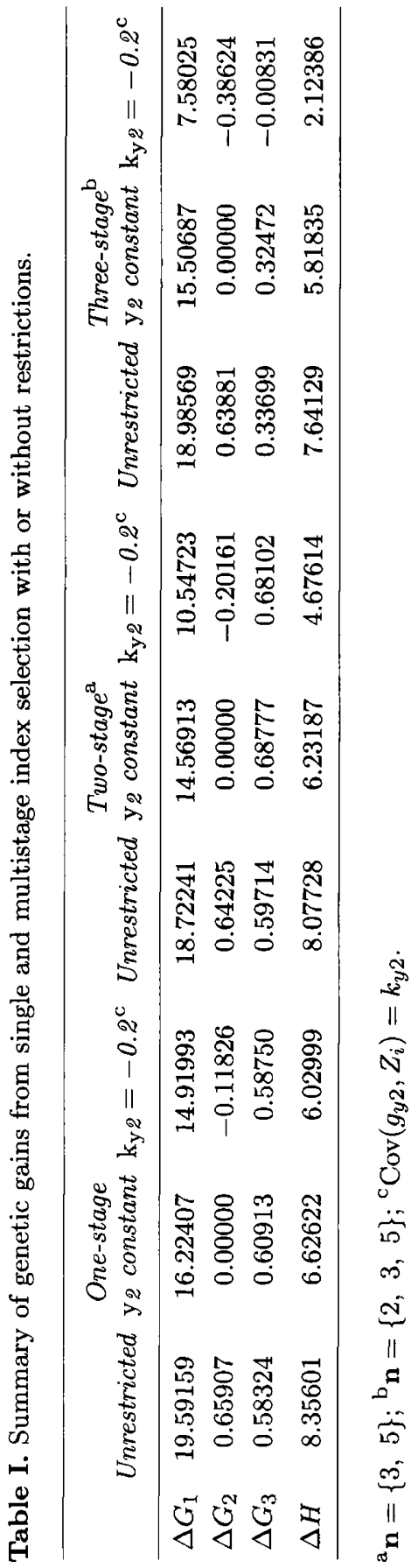


knowledge of economic values is vague, especially over the long term (Pešek and Baker, 1969; Niebel and Van Vleck, 1982; Itoh and Yamada, 1988a, b). Under these circumstances, it may be more efficient to give relative values or to assign predetermined amounts of genetic gains to the traits (Tallis, 1962; Itoh and Yamada, $1988 \mathrm{a}, \mathrm{b})$.

The coefficients for the restricted index presented in this note are found so that the correlation between $Z_{i}$ and $H$ at each stage is maximum with constraints $\operatorname{Cov}\left(Z_{i}, Z_{j}\right)=0$ for $i \neq j$. An index constructed in that manner will have explicitly determined solutions for the truncation points. However, genetic gain will be somewhat less than that obtained without constraints (Xu and Muir, 1992). But Xu and Muir (1991) also showed that under certain conditions the efficiency of restricted multistage index selection may greatly exceed that of conventional multistage selection because the latter dose not incorporate information from previous stages of selection into the current stage. Owing to computational advantages over best linear unbiased prediction (BLUP) under selection models, this procedure can be used to effectively design performance testing and selection programs that will optimize profit in commercial breeding (Xu et al, 1995). For situations with phenotypic variances and covariances, heritabilities, economic values, and costs of measurement known, the breeder can investigate many options relative to choices of traits and stages with respect to maximizing either total economic value of genetic change or profit.

\section{ACKNOWLEDGMENTS}

The authors would like to thank the referees for helpful comments in an early draft. This research was supported by the National Research Initiative Competitive Grants Program/USDA 95-37205-2313 to SX.

\section{REFERENCES}

Brascamp EW (1984) Selection indices with constraints. Anim Breed Abst 52, 645-654

Cunningham EP (1975) Multi-stage index selection. Theor Appl Genet 46, 55-61

Ducrocq V, Colleau JJ (1989) Optimum truncation points for independent culling level selection on a multivariate normal distribution, with an application to dairy cattle selection. Genet Sel Evol 21, 185-198

Harville DA (1975) Index selection with proportionality constraints. Biometrics 31, 223-225

Hazel LN (1943) The genetic basis for constructing selection indexes. Genetics 28, 476-490

Itoh Y, Yamada Y (1988a) Linear selection indices for non-linear profit functions. Theor Appl Genet 75, 553-560

Itoh Y, Yamada Y (1988b) Selection indices for desired relative genetic gains with inequality constraints. Theor Appl Genet 75, 731-735

James JW (1968) Index selection with restrictions. Biometrics 24, 1015-1018

Kempthorne O, Nordskog AW (1959) Restricted selection indices. Biometrics 15, 10-19

Lin CY (1989) A united procedure of computing restricted best linear unbiased prediction and restricted selection index. J Anim Breed Genet 107, 311-316

Namkoong G (1970) Optimal allocation of selection intensity in two stages of truncation selections. Biometrics 26, 465-576 
Niebel E, Van Vleck LD (1982) Selection with restriction in cattle. J Anim Sci 55, 439-453 Norell L, Arnason T, Hugason K ( 1991) Multistage index selection in finite populations. Biometrics 47, 205-221

Pešek J, Baker RJ (1969) Desired improvement in relation to selection indices. Can J Plant Sci 49, 803-804

Rao CR (1962) Problems of selection with restrictions. $J R$ Statist Soc B 24, 401-405

Smith HF (1936) A discriminant function for plant selection. Ann Eugen 7, 240-250

Tallis GM (1962) A selection index for optimum genotype. Biometrics 18, 120-122

Tallis GM (1968) Selection for an optimum growth curve. Biometrics 24, 169-177

Xu S, Muir WM (1991) Multistage selection for genetic gain by orthogonal transformation. Genetics 129, 963-974

$\mathrm{Xu}$ S, Muir WM (1992) Selection index updating. Theor Appl Genet 83, 451-458

Xu S, Martin TG, Muir WM (1995) Multistage selection for maximum return with an application to beef cattle breeding. J Anim Sci 73, 699-710

Young SSY (1964) Multi-stage selection for genetic gain. Heredity 19, 131-145

Young SSY, Weiler H (1960) Selection for two correlated traits by independent culling levels. J Genet 57, 329-338 